\title{
Estudios de zooplancton marino en Costa Rica: una revisión y perspectivas a futuro
}

\author{
Álvaro Morales-Ramírez ${ }^{1,2}$, Marco Corrales-Ugalde ${ }^{1,3}$, Octavio Esquivel-Garrote ${ }^{4}$, Allan Carrillo- \\ Baltodano $^{5}$, Karina Rodríguez-Sáenz ${ }^{6} \&$ Carolina Sheridan ${ }^{2}$ \\ 1. Centro de Investigación en Ciencias del Mar y Limnología (CIMAR), Universidad de Costa Rica, San Pedro, 11501- \\ 2060 San José, Costa Rica; alvaro.morales@ucr.ac.cr \\ 2. Escuela de Biología, Universidad de Costa Rica, 2060 San José, Costa Rica; csheridanrodriguez@gmail.com \\ 3. Oregon Institute of Marine Biology, University of Oregon, Eugene, OR 97403, USA; mcugalde88@gmail.com \\ 4. Laboratorio de zooplâncton, Instituto de Oceanografia Biológica, Universidade Federal do Rio Grande, Campus \\ Carreiros, 96201-900, Avenida Itália km 8, Rio Grande, RS, Brasil; octageo@gmail.com \\ 5. Biology Department, Clark University, 950 Main Street, Worcester, MA 01610, USA; acarrillobaltodano@clarku.edu \\ 6. Grupo Hábitat, San José, Costa Rica; karinarodriguez@racsa.co.cr
}

Recibido 04-VII-2017. Corregido 08-I-2018. Aceptado 25-I-2018.

\begin{abstract}
Marine zooplankton studies in Costa Rica: a review and future perspectives. This review summarizes marine zooplankton research performed in the Costa Rican Pacific and Caribbean coasts and at Isla del Coco National Park. Composition, abundance, biomass, distribution and some ecological features of the zooplankton for each system is discussed. Pacific coastal zooplankton composition has been described in Bahía Salinas and Bahía Culebra. The local oceanography of these bays is heavily influenced by the Papagayo wind jet, which produces seasonal coastal upwelling and most likely drives the seasonal changes in zooplankton abundance and biomass. Copepods and ostracods were the dominant taxonomic groups throughout the year with a strong seasonality, furthermore 53 hydromedusae species has been found, where Liriope tetraphylla (Chamisso \& Eysenhardt, 1821), Solmundella bitentaculata (Quoy \& Gaimard, 1833) and Aglaura hemistoma (Péron \& Lesueur, 1810), were the most abundant species. Zooplankton herbivory rates at Gulf of Nicoya estuary was estimated over $50 \%$ removal of primary production. This is a typical value for tropical productive environments. Copepods numerically dominated zooplankton community from the Gulf of Nicoya. Other dominant taxa includes merozooplanktonic decapod larvae and ichthyoplankton. Biomass is high and there are strong crossshelf gradients in the Gulf. Zooplankton collected in the southern Pacific coast (close to Isla del Caño) is also dominated by copepods, while Bahía Coronado has a mixed zooplankton composition of oceanic and coastal transition waters. In Golfo Dulce, zooplankton is highly diverse and dominated by copepods, appendicularians, and ostracods. About $35 \%$ of daily primary production is consumed by microzooplankton, and zooplankton adapt to anomalous conditions of El Niño-Southern Oscillation (ENSO). Coco's Island, and other oceanic islands in the Eastern Tropical Pacific are "hot spot" of zooplankton diversity, where > 160 zooplankton species have been reported (predominantly copepods). The biomass is dominated by mesozooplankton, and new species records for the Eastern Tropical Pacific have been found in this insular ecosystem. In the other hand, early works of 1980 carried out at Caribbean coast (Cahuita National Park), show a considerably low zooplankton diversity and abundance while research at the end of the 2000 decade recognized a significant increase in zooplankton diversity, specially fish larvae. Recent zooplankton studies have added six new copepod species and several new records of species from different taxonomic groups such as appendicularians, amphipods, chaetognats, euphausiids, gastropods, and polychaetes. Future work should focus on monthly long-term monitoring programs to investigate the effects of ocean acidification and the trophic dynamics associated with fisheries. Alike, longterm studies are needed to see trends of change in planktonic communities, especially comparing areas under human activity in the coastal zone (tourism, port, urban development) with protected areas as targets for study. An increase of sampling efforts must be done in the relatively understudied Northern Caribbean coast. Rev. Biol. Trop. 66(Suppl. 1): S24-S41. Epub 2018 April 01.
\end{abstract}

Key words: abundance, biomass, ecology, vertical migration, composition, Tropical East Pacific. 
El zooplancton marino comprende organismos productores secundarios o terciarios que forman parte del plancton, que junto con el fitoplancton forman la base de las redes alimentarias en los océanos. Según sus estrategias de vida, se clasifica en holozooplancton (ciclo de vida completo en el plancton, como microcrustáceos, apendicularias, salpas, quetgnatos) y el merozooplancton (parte de ciclo de vida en el plancton, como larvas de invertebrados bénticos y peces). Los estudios del zooplancton en los últimos 25-30 años han permitido comprender de una manera más integral algunos procesos ecológicos pelágicos, desde el análisis de la diversidad marina con criterios morfológicos y moleculares, la estructura comunitaria, las relaciones tróficas, la función ecológica de las especies pequeñas, la micro-distribución, aspectos reproductivos y los cambios poblacionales y fisiológicas del zooplancton en respuesta a El Niño-Oscilación Sur (ENSO) y el cambio global, incluyendo su modulación en el flujo de partículas y ciclo del carbono (Paffenhöfer, Hundley, \& Davis, 1989; Bathmann et al., 2001). Una característica relevante del zooplancton marino es su biodiversidad como indicador de la salud del ecosistema, ya que evidencia la dinámica y características generales de las interacciones biológicas en la comunidad (competencia, depredación, parasitismo, reclutamiento y productividad) (Mittelbach et al., 2001). La posible disminución de la diversidad marina ha generado esfuerzos recientes para identificar su importancia en el funcionamiento de los ecosistemas (Pfisterer $\&$ Schmith, 2002). A pesar de que se ha generado un enorme conocimiento ecológico del zooplancton marino en diferentes regiones del mundo como en altas latitudes del hemisferio norte, existen regiones en el mundo donde aún no se conocen aspectos básicos de la composición, abundancia, biomasa y diversidad del zooplancton, especialmente en ambientes poco estudiados como islas oceánicas y en latitudes tropicales (Morales-Ramírez, 2008).

Los primeros estudios del zooplancton marinos de Costa Rica iniciaron con la ecología larval (merozooplancton) de crustáceos en el Golfo de Nicoya (Epifanio \& Dittel, 1982, 1984) y la caracterización de estrategias de dispersión (Wehrtmann \& Dittel, 1990). Los primeros estudios del holozooplancton marino se realizaron en la Isla del Caño (Guzmán \& Obando, 1988) y en aguas del Parque Nacional Cahuita (Morales-Ramírez \& Murillo, 1996), posteriormente durante la expedición del Victor Hensen (Wolff \& Vargas, 1994), se publicaron notas ecológicas sobre el zooplancton en el Golfo Dulce y Golfo de Nicoya (Hossfeld, Molina-Ureña, \& Morales-Ramírez, 1994; von Wangelin \& Wolff 1996) y trabajos sobre la abundancia, biomasa, composición y distribución del zooplancton marino en la costa del Pacífico costarricense (Hossfeld et al., 1994). Otros estudios más recientes han evaluado el impacto de El Niño-Oscilación Sur de 19971998 en la dinámica del zooplancton del Golfo de Nicoya (Morales-Ramírez \& BrugnoliOlivera, 2001) y del Golfo Dulce (MoralesRamírez \& Nowaczyk, 2006; Quesada-Alpízar \& Morales-Ramírez, 2006; Morales-Ramírez \& Jacob, 2008). Posteriormente, se evaluó la dinámica trófica del zooplancton en el Golfo de Nicoya (Brugnoli-Olivera \& Morales-Ramírez, 2008) y el Golfo Dulce (Estrada, 2003).

En estudios subsecuentes realizados durante los años noventa, se caracterizó la estructura del zooplancton costero entre Moín y el aeropuerto de Limón, localizado en el Caribe costarricense (Morales-Ramírez, 1997). La abundancia y biomasa del macrozooplancton y el mesozooplancton fue estudiada durante el periodo de La Niña-Oscilación Sur 1999-2000 en Bahía Culebra, Bahía Salinas y en el Golfo de Santa Elena (Bednarski \& Morales-Ramírez, 2004; Rodríguez \& Morales-Ramírez, 2012; Rodríguez-Saénz, Vargas-Zamora, \& SeguraPuertas, 2012).

El zooplancton oceánico del Parque Nacional de la Isla del Coco también ha sido ampliamente estudiado (Morales-Ramírez, 2008; Castellanos-Osorio, Hernández-Flores, Morales-Ramírez, \& Corrales-Ugalde, 2012; Gasca \& Morales-Ramírez, 2012; JiménezCueto, Suárez-Morales, \& Morales-Ramírez, 2012; Suárez \& Gasca, 2012; Azofeifa-Solano, 
Corrales-Ugalde, Castellanos-Osorio, \& Morales-Ramírez, 2016; Morales-Ramírez, Corrales-Ugalde, Esquivel-Garrote, \& SuárezMorales, en prep.).

El libro sobre Biodiversidad Marina de Costa Rica (Wehrtmann \& Cortés, 2009) reanudó el interés en los estudios sobre la diversidad taxonómica del plancton, logrando el mejor inventario de la diversidad del zooplancton hasta la fecha para Costa Rica hasta finales de la primera década del siglo XXI.

El objetivo de este trabajo es realizar una revisión comprensiva sobre el desarrollo de la investigación en zooplancton marino en los diferentes hábitats costeros de Costa Rica (costa Pacífica y del Caribe) y en hábitats insulares oceánicos del Parque Nacional Isla del
Coco ubicada a $532 \mathrm{~km}$ de la costa Pacífica, y se reflexiona sobre el tipo de estudios futuros necesarios en zooplancton para la gestión integrada de los recursos marinos de las costas de Costa Rica.

\section{RESULTADOS Y DISCUSIÓN}

Las áreas de muestreo de zooplancton marino en aguas costarricense realizadas entre 1982 y 2012 se muestran en la figura 1. Es notorio que el mayor esfuerzo de muestreo e investigación se ha realizado en la costa del Pacífico en comparación con la costa del Caribe. El cuadro 1 sintetiza la información de los tipos de redes de zooplancton y tamaños de malla utilizados, el tipo de muestreo y la profundidad

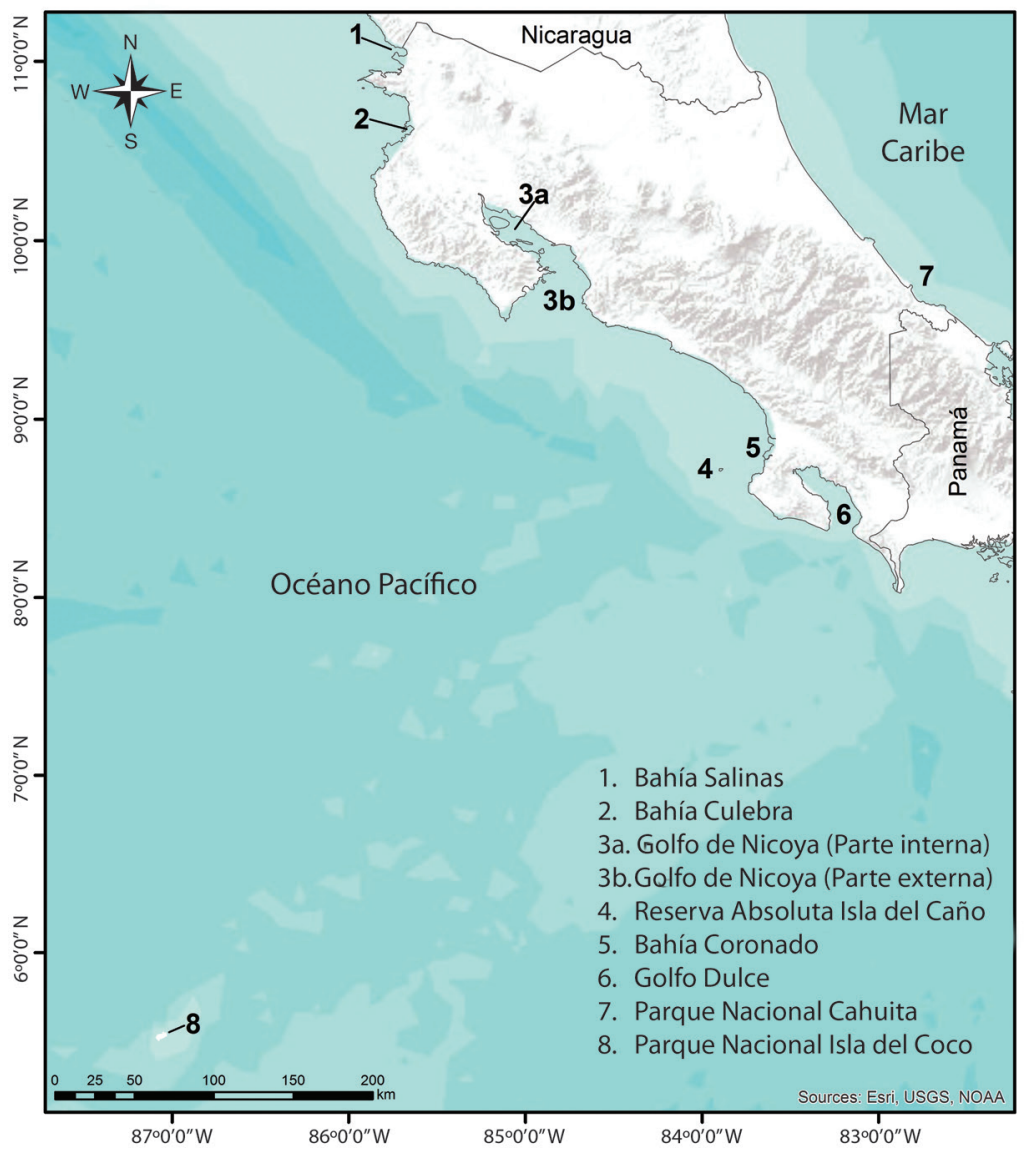

Fig. 1. Sitios de muestreo del zooplancton marino en los ambientes costeros y oceánicos de Costa Rica.

Fig. 1. Sampling sites for marine zooplankton in coastal and oceanic environments of Costa Rica. 
CUADRO 1

Aspectos metodológicos de muestreo de estudios del holozooplancton marino en Costa Rica

TABLE 1

Methodological aspects of sampling of marine holozooplankton studies in Costa Rica

\begin{tabular}{|c|c|c|c|c|}
\hline Sitio de estudio & Red y poro $(\mu \mathrm{m})$ & $\begin{array}{l}\text { Tipo de muestreo } \\
\text { (H:horizontal, } \\
\text { V:vertical) }\end{array}$ & $\begin{array}{l}\text { Determinación Biomasa } \\
\text { (PS: peso seco, } \mathrm{PH} \text { : } \\
\text { peso húmedo) }\end{array}$ & Referencia \\
\hline Golfo de Nicoya & $200-280-300 \mu \mathrm{m}$ & $\mathrm{H} \mathrm{y} \mathrm{V}$ & PS, PH & $\begin{array}{l}\text { Hossfeld et al. (1994); von Wangelin \& Wolff } \\
\text { (1996); Morales-Ramírez (1996); Hossfeld } \\
\text { (1996), Morales-Ramírez \& Brugnoli-Olivera } \\
\text { (2001); Brugnoli-Olivera et al. (2004), Brugnoli- } \\
\text { Olivera \& Morales-Ramírez (2008) }\end{array}$ \\
\hline Golfo Dulce & $180-200-500 \mu \mathrm{m}$ & $\mathrm{H} \mathrm{y} \mathrm{V}$ & $\mathrm{H} \mathrm{y} \mathrm{V}$ & $\begin{array}{l}\text { Jacob (1996); Nowaczyk (1998), Quesada- } \\
\text { Alpízar (2001); Estrada (2003); Quesada-Alpízar } \\
\text { \& Morales-Ramírez (2004); Morales-Ramírez } \\
\text { \& Jacob (2008); Morales-Ramírez \& Nowaczyk } \\
\text { (2006); Morales-Ramírez, Acuña-González, } \\
\text { Lizano, Alfaro \& Gómez (2015) }\end{array}$ \\
\hline $\begin{array}{l}\text { Golfo Papagayo- } \\
\text { Bahía Culebra }\end{array}$ & $200-500-1000 \mu \mathrm{m}$ & V & PS & $\begin{array}{l}\text { Bednarski (2001); Bednarski \& Morales-Ramírez } \\
\text { (2004); Rodríguez-Sáenz (2005); Rodríguez- } \\
\text { Sáenz \& Morales-Ramírez (2012) }\end{array}$ \\
\hline Bahía Salinas & $200 \mu \mathrm{m}$ & $\mathrm{V}$ & PS & Morales-Ramírez (2015) \\
\hline $\begin{array}{l}\text { Parque Nacional } \\
\text { Cahuita }\end{array}$ & $280 \mu \mathrm{m}$ & $\mathrm{H}$ & & $\begin{array}{l}\text { Morales (1987); Morales-Ramírez \& Murillo } \\
\text { (1996); Morales-Ramírez (1997, 2001); } \\
\text { Carrillo (2012) }\end{array}$ \\
\hline $\begin{array}{l}\text { Parque Nacional } \\
\text { Isla del Coco }\end{array}$ & 200-280- $500 \mu \mathrm{m}$ & $\mathrm{H} \mathrm{y} \mathrm{V}$ & PS & $\begin{array}{l}\text { Morales-Ramírez (2008); } \\
\text { Morales-Ramírez \& Montero Vargas (2014) }\end{array}$ \\
\hline
\end{tabular}

o intervalos de muestreo, la técnica de conteo y determinación de biomasa utilizada, así como los estudios publicados con cada muestreo, el cuadro 2 resume las mediciones de biomasa seca y abundancia del zooplancton reportadas en diferentes ambientes de Costa Rica.

\section{Estudios Ecológicos en la Costa Pacífica de Costa Rica}

Golfo de Nicoya: Los primeros estudios compararon el merozooplancton del Golfo de Nicoya con un estuario de clima templado, específicamente la bahía de Chesapeake en la costa de Atlántico de Estados Unidos de Norte América (Epifiano \& Dittel, 1982). En el Golfo de Nicoya se evidenció una clara diferencia en la composición de larvas de decápodos entre la parte interna del Golfo y la parte externa: especímenes de las familias Ocypodidae, Pinnotheridae y Xanthidae fueron más comunes en aguas internas, mientras que de las familias Majidae y Portunidae fueron más comunes en la parte externa del Golfo. Asimismo la abundancia de larvas de decápodos braquiuros cambia con los ciclos de marea y las estaciones de seca y lluviosa (Epifanio \& Dittel, 1984; Dittel \& Epifanio, 1990). Estas diferencias en abundancia se asociaron a la dinámica oceanográfica y composición del substrato debido a que la parte más interna y somera de la bahía, el fondo tiene predominantemente sedimentos finos (Maurer \& Vargas, 1984). Por otro lado, la parte externa está influenciada por el frente salino cerca de las Islas Negritos y por esta razón tiene mayor influencia oceánica (Voohris, Epifanio, Maurer, Dittel, \& Vargas, 1983). Por otra parte, Wehrtmann \& Dittel (1990) demostraron el transporte de larvas de decápodos asociados a hojas de manglar en la parte interna del Golfo como una estrategia de dispersión. 
El primer estudio de copépodos de la parte interna del Golfo de Nicoya reportó 12 especies típicas de ambientes estuarinos (Paracalanus parvus (Claus, 1863), Acartia lilljeborgii (Giesbrecht, 1889), Pseudodiaptomus wrighti (Johnson , 1964), Pseudodiaptomus panamensis (Walter, 1989) , Euterpina acutifrons (Dana, 1847) y Hemicyclops thalassius (Vervoort \& Ramírez, 1966)) (Morales-Ramírez \& Vargas, 1995). La expedición del Víctor Hensen llevada a cabo en conjunto entre la Universidad de Costa Rica y el Centro Leibniz de Ecología Marina Tropical (ZMT) de la Universidad de Bremen, Alemania (dic. 1993-feb. 1994), incrementó el listado taxonómico del golfo a 37 (Morales-Ramírez, 1996). Existe una evidente diferencia en la composición de especies en las aguas cercanas a la desembocadura del río Tempisque dominada por especies estuarinas y las estaciones externas dominadas por especies oceánicas como Oncaea venusta (Philippi, 1843), Pareucalanus attenuatus (Dana, 1849), Eucalanus elongatus (Dana, 1848), Rhincalanus nasutus (Giesbrecht, 1888) y Pleuromamma robusta (Dahl F., 1893) (Morales-Ramírez, 1996).

Estudios sobre biomasa del zooplancton en el Golfo de Nicoya son escasos. von Wangelin \& Wolff (1996) realizaron la primera estimación de la biomasa del zooplancton mediante

\section{CUADRO 2}

Ámbito de biomasa seca y abundancia reportados para las comunidades zoopláncticas estudiadas en Costa Rica

TABLE 2

Range of dry biomass and abundance reported for the zooplanktonic communities studied in Costa Rica

\begin{tabular}{|c|c|c|c|c|c|}
\hline Sitio & $\begin{array}{l}\text { Años de } \\
\text { muestreo }\end{array}$ & $\begin{array}{l}\text { Fracción de } \\
\text { tamaño }(\mu \mathrm{m})\end{array}$ & $\begin{array}{l}\text { Biomasa } \\
\left(\mathrm{mg} \mathrm{PS} / \mathrm{m}^{3}\right)\end{array}$ & $\begin{array}{l}\text { Abundancia } \\
\left.\text { (org. } / \mathrm{m}^{3}\right)\end{array}$ & Referencia \\
\hline Bahía Salinas & 2011-14 & $>200$ & $18.4-86.2$ & $1649->135000$ & Morales-Ramírez (2015) \\
\hline \multirow[t]{2}{*}{ Bahía Culebra } & 1999-2001 & $>200$ & $5.14-178.40$ & $3969-6216$ & $\begin{array}{l}\text { Rodríguez-Sáenz (2006); Rodríguez- } \\
\text { Saénz \& Morales-Ramírez (2012) }\end{array}$ \\
\hline & & $>500$ & until 1066 & $9813-54286$ & $\begin{array}{l}\text { Bednarski \& Morales-Ramírez (2004); } \\
\text { Morales-Ramírez, datos sin publicar }\end{array}$ \\
\hline \multirow[t]{3}{*}{ Golfo de Nicoya } & 1994 & $\begin{array}{l}280-500 \\
500-1000 \\
1000-2000\end{array}$ & $\begin{array}{l}169-79 \\
259.6-106.0 \\
129.9-65.6\end{array}$ & & Morales-Ramírez, datos sin publicar \\
\hline & 1995 & $\begin{array}{l}280-500 \\
500-1000 \\
1000-2000\end{array}$ & $\begin{array}{l}80.0-37.6 \\
237.4-98.8 \\
198.1-71.8\end{array}$ & $1458-9141$ & \\
\hline & 2001 & $>200$ & & & Brugnoli-Olivera et al. (2004) \\
\hline \multirow[t]{2}{*}{ Golfo Dulce } & $1997-98$ & $>153$ & $25.0-60.0$ & $946-9921$ & $\begin{array}{l}\text { Quesada-Alpízar (2001); Quesada- } \\
\text { Alpízar \& Morales-Ramírez (2004) }\end{array}$ \\
\hline & $2005-07$ & $>200$ & $3.6-154.2$ & $<500-25000$ & Morales-Ramírez et al. (2015) \\
\hline \multirow[t]{2}{*}{$\begin{array}{l}\text { Parque Nacional } \\
\text { Isla del Coco }\end{array}$} & $2010-12$ & $\begin{array}{l}>200 \\
>500\end{array}$ & $\begin{array}{l}45.0-153.0 \\
14.0-68.9\end{array}$ & $\begin{array}{l}40-445 \\
2-164\end{array}$ & $\begin{array}{l}\text { Morales-Ramírez \& Montero-Vargas } \\
\text { (2014) }\end{array}$ \\
\hline & $2010-12$ & $\begin{array}{l}>200 \\
>500\end{array}$ & $5.7-50.0$ & $223-19$ & Esquivel-Garrote (2015) \\
\hline \multirow{2}{*}{$\begin{array}{l}\text { Parque Nacional } \\
\text { Cahuita }\end{array}$} & 1984 & $>200$ & & $2449-4635$ & Morales-Ramírez \& Murillo (1996) \\
\hline & $2010-11$ & $>200$ & $0.49-85.87$ (seston) & $1145-112422$ & $\begin{array}{l}\text { Carrillo Baltodano \& Morales-Ramírez } \\
\text { (2016) }\end{array}$ \\
\hline
\end{tabular}


un análisis espectral con base a tamaño logarítmico en las estaciones internas y externas del Golfo durante la transición de la época lluviosa de 1993 y la seca de 1994. El espectro de biomasa en las estaciones internas al final de la época lluviosa fue dominado de manera discontinua por las especies pequeñas del zooplancton, mientras que durante la época seca fue dominada por especies de mayor tamaño de manera más continua. Las variaciones estacionales en la región externa del Golfo de Nicoya tienen gradientes de biomasas menos pronunciadas, espectro de biomasa y composición de especies, ya que la pendiente de biomasa fue relativamente más continua y menor por la ausencia de grandes depredadores. Hossfeld (1996) realizó un análisis de la distribución y biomasa de los quetognatos del Golfo de Nicoya como para el Golfo Dulce, encontrando especies de los géneros Sagitta y Kronhitta, con un gradiente decreciente en la riqueza de especies de la región más externa (ocho especies) a la región más interna (una especie). Los quetogantos costeros fueron dominados por juveniles y adultos de Parasagitta friderici (Ritter-Záhony, 1911) en el Golfo de Nicoya.

La biomasa del mesozooplancton se estudió en siete estaciones internas del Golfo de Nicoya durante la mitad de la década de los noventa (Morales-Ramírez, datos sin publicar). La biomasa seca del mesozooplancton fue mayor en la fracción 500-1000 $\mu \mathrm{m}$ (Cuadro 2) con un ámbito de 106-260 mg $\mathrm{PS} / \mathrm{m}^{3}$ durante 1994 y $99-237 \mathrm{mg} \mathrm{PS} / \mathrm{m}^{3}$ durante 1995 . Estos ámbitos de biomasa demuestran que el Golfo de Nicoya uno de los estuarios tropicales más productivos del mundo (Cloern, Foster, \& Kleckner, 2014). Debido a la elevada productividad biológica en la región interna del Golfo de Nicoya, también fue investigado el impacto de El Niño-Oscilación del Sur (ENSO) 19971998 en la estacionalidad de la disponibilidad de fitoplancton (Morales-Ramírez \& BrugnoliOlivera, 2001) que provocó un aumento en la biomasa del zooplancton dominado por tres especies de copépodos herbívoros (A. lilljeborgii, Pseudodiaptomus spp. y P. parvus). El $57.6 \%$ de la producción primaria en el Golfo de Nicoya fue consumido por el zooplancton herbívoro (Brugnoli-Olivera \& MoralesRamírez, 2008). Esto es una tasa de herbivoría ligeramente mayor que el $47 \%$ encontrado en el Pacífico ecuatorial (Zhang, Dam, White, \& Roman, 1995).

La abundancia del mesozooplancton en la parte interna del Golfo varía de 1458 a 9142 org./ $\mathrm{m}^{3}$ con un mayor aporte de los copépodos $(\sim 80 \%)$ y quetognatos $(16 \%)$ dominando el holozooplancton. Las larvas de crustáceos contribuyeron con el $66 \%$ y el ictioplancton con el $18 \%$ de la composición total del merozooplancton (Brugnoli-Olivera, Díaz-Ferguson, Delfino-Machin, Morales-Ramírez, \& Dominici-Arosanema, 2004).

Isla del Caño y Bahía Coronado: En la Isla del Caño se estudió la composición del zooplancton asociado a arrecifes coralinos. Los copépodos constituyeron entre 40-70\% de la abundancia total. También se observó que todos los grupos incrementan la abundancia de zooplancton por la noche y algunos como isópodos y poliquetos solo aparecieron de noche, y se resalta el carácter aparentemente estacional de las abundancias del zooplancton en este sitio (Guzmán \& Obando, 1988). Morales-Ramírez (2001) reveló una composición mixta de especies oceánicas de copépodos en Bahía Coronado (Neocalanus gracilis (Dana, 1852), Euchaeta longicornis (Giesbrecht, 1888), Pareucalanus attenuatus (Dana, 1849) y Haloptilus ornatus (Giesbrecht, 1893) y especies neríticas (Clausocalanus furcatus (Brady, 1883) y Clausocalanus pergens (Farran, 1926) y Paracalanus parvus).

Golfo Dulce: Los estudios de zooplancton en el Golfo Dulce son más recientes. Hasta el momento se han reportado 36 especies de copépodos en el Golfo Dulce. Esta riqueza específica es alta comparada con otras regiones costeras de Costa Rica debido a la presencia de especies tanto neríticas como oceánicas en el Golfo (Morales-Ramírez, 1996). von Wangelin \& Wolff (1996) estimaron la biomasa del zooplancton con la misma metodología utilizada 
en el Golfo de Nicoya. Las zonas costeras del Golfo Dulce mostraron las mismas tendencias que en el Golfo de Nicoya, con excepción de que en la época seca el zooplancton gelatinoso fue considerablemente abundante (von Wangelin \& Woff, 1996).

La abundancia y biomasa del zooplancton en el Golfo Dulce son notablemente inferiores que en el Golfo de Nicoya y estas diferencias fueron más pronunciadas durante la época de lluvia (von Wangelin \& Wolff, 1996). Los cambios de la biomasa, abundancia y composición del zooplancton de la época lluviosa a época seca en las oceánicas fueron menos pronunciados. A diferencia del Golfo de Nicoya, la pendiente del espectro de biomasa $\left(\log _{10}\right)$ del Golfo Dulce fue más pronunciada y continua. Esto sugiere relativamente mejores condiciones trofodinámicas (balance energético) que permiten un mayor desarrollo de la comunidad del zooplancton en términos de abundancia y biomasa. Los juveniles y adultos del quetognato Parasagitta popovicii (Sund, 1961) fueron los grupos más abundantes en las estaciones internas del Golfo Dulce. Sin embargo, el espectro de biomasa fue más continuo y de mayor ámbito geográfico. Esta especie de quetognato puede estructurar la comunidad de plancton por una fuerte presión de depredación (Hossfeld, 1996).

Quesada-Alpizar \& Morales-Ramírez (2006) analizaron la composición, abundancia, biomasa y distribución vertical del mesozooplancton bajo condiciones del ENSO. La mayor abundancia y biomasa dominada por la fracción de zooplancton pequeño $(153-500 \mu \mathrm{m})$ se encontró en el estrato superior por encima de la termoclina (Cuadro 2). Las abundancias llegaron hasta $9921 \mathrm{org} . / \mathrm{m}^{3}$ en el estrato superior y descendieron abruptamente a 946 org./ $\mathrm{m}^{3}$ en el estrato por debajo de la termoclina. Esta disminución drástica coincide con el típico gradiente de disminución de la concentración de oxígeno por debajo de la termoclina del Golfo (Quesada-Alpízar \& Morales-Ramírez, 2004). La biomasa fue mayor en el estrato superior (25-60 mg PS $\left./ \mathrm{m}^{3}\right)$. Diez años después se muestrearon las mismas estaciones confirmando que las mayores abundancias y biomasas se encuentran en el estrato superior (Morales-Ramírez et al., 2015). A diferencia del primer estudio, en el segundo estudio se realizaron muestreos horizontales mostrando que las abundancias y biomasas secas fueron considerablemente superiores a las del perfil vertical $\left(25000 \mathrm{org} . / \mathrm{m}^{3}\right)$. Esta alta abundancia coincidió con el mayor valor de biomasa seca $154 \mathrm{mg} \mathrm{PS} / \mathrm{m}^{-3}$. Este es un valor sumamente alto considerando el carácter mesotrófico del golfo (Morales-Ramírez et al., 2015) y en comparación con el Golfo de Nicoya registrado durante la década de los 1990's (Cuadro 2). En ambos trabajos, las especies de copépodos pequeñas del Orden Cyclopoida de los géneros Oncaea, Corycaeus y Oithona, contribuyeron entre el 44-83.6\% de la abundancia total. Otros grupos abundantes fueron las apendicularias y ostrácodos (Morales-Ramírez et al., 2015).

Una investigación durante la transición de la época lluviosa a la seca (1997-1998) y en presencia de ENSO, permitió el registro de 41 especies de zooplancton gelatinosos (17 hidromedusas y 16 sifonóforos, dos de ctenóforos y seis especies de salpas), donde se analizó las diferencias espaciales y temporales en su composición y abundancia (Morales-Ramírez \& Nowaczyk, 2006). La diversidad y abundancia de organismos gelatinosos aumentaron de diciembre 1997 a febrero 1998, con un gradiente espacial de mayor diversidad y abundancia en estaciones en medio y en la parte externas del Golfo Dulce disminuyendo estas hacia las estaciones internas. Dichas diferencias probablemente son el reflejo de respuestas poblacionales a las condiciones del ENSO 1997-1998. Quesada-Alpízar \& Morales-Ramírez (2004) reportaron durante dos años entradas de aguas oceánicas, frías, de altas salinidades y ricas en nutrimentos. Estas condiciones promueven una mayor productividad primaria en la parte interna del Golfo. Este comportamiento del zooplancton en el Golfo Dulce parece ser lo suficientemente variable (plástico) para adaptarse a condiciones anómalas como el ENSO (Morales-Ramírez et al., 2015). 
Otros estudios en Golfo Dulce analizaron la abundancia, biomasa, distribución vertical y relaciones biométricas de cuatro especies de ostrácodos: Cypridina americana (Müller, 1890), Euconchoecia chierchiae (G.W. Müller, 1890), Porroecia spinirostris (Claus, 1874) y Porroecia spp. Las relaciones biométricas de las primeras dos especies revelaron diferencias significativas de longitud entre estadíos de desarrollo dependiendo de la estación del año y demostrando un dimorfismo sexual entre machos y hembras. La biomasa tuvo significativamente mayores valores en septiembre (8 $\mathrm{mg} \mathrm{PS} / \mathrm{m}^{3}$ ) que en mayo $\left(3 \mathrm{mg} \mathrm{PS} / \mathrm{m}^{3}\right)$, a pesar de que no se encontraron diferencias estadísticas en la abundancia de esos meses (700 vs 880 org. $\left./ \mathrm{m}^{3}\right)$. C. americana y E. chierchiae tuvieron una alternancia en la abundancia y dominancia entre día/noche. Todos los estadios de ambas especies se distribuyeron principalmente en los primeros $20 \mathrm{~m}$ de profundidad, excepto E. chierchiae, que se mantuvo siempre por debajo de los $20 \mathrm{~m}$ (Morales-Ramírez \& Jacob, 2008). Este "particionamiento" de hábitat ha sido previamente documentada en copépodos oceánicos (Williams, 1991) y podría ser una estrategia ontogenética para C. americana. Resalta la extraordinaria abundancia de ostrácodos en el Golfo Dulce y su relevancia trófica en el ecosistema pelágico. Cypridina americana es una especie detritívora y depredadora facultativa y E. chierchiae se alimenta principalmente de materia orgánica (Jacob, 1996; Morales-Ramírez \& Jacob, 2008).

Para tener una mejor idea de la dinámica trófica del Golfo Dulce se realizaron experimentos de tasas de alimentación mediante incubaciones en laboratorio de los copépodos P. parvus, Acartia sp., Centropages furcatus (Dana, 1849) y el ostrácodo C. americana (Estrada, 2003). Un análisis multivariado (Análisis Múltiple Discriminante - AMD) mostró que la clorofila aumentó en las incubaciones con $C$. americana y mantuvo valores similares a los controles en las incubaciones con Acartia sp. Por otra parte, C. furcatus mantuvo niveles de clorofila más altos que los controles en la mayoría de los casos. Únicamente $P$. parvus disminuyó la concentración de clorofila de las botellas en casi todos los casos. Esto sugiere que Acartia sp y $C$. furcatus no contribuyen a la herbivoría del sistema (Estrada, 2003). Más recientemente se ha analizado la contribución directa e indirecta del zooplancton en la pérdida de nitrógeno fijado en el Golfo Dulce donde prevalece un ambiente con concentraciones bajas de oxígeno a poca profundidad (Stief, Lundgaard, Morales-Ramírez, Thamdrup, \& Glud, 2017). El zooplancton parece habitar ambientes anóxicos en la zona epipelágica. Experimentos con altos contenidos del isótopo estable ${ }^{15} \mathrm{~N}$ revelaron que bajo condiciones de alta abundancia de copépodos muertos y ostrácodos (vivos y muertos), el zooplancton influye procesos en el ciclo del nitrógeno aeróbico (nitrificación) y anaeróbico (desnitrificación y reducción disimilatoria del nitrato a amonio). El enriquecimiento de amonio $\left(\mathrm{NH}_{4}^{+}\right)$, nitrógeno y óxido nitroso $\left(\mathrm{N}_{2} \mathrm{O}\right)$ producido por copépodos y ostrácodos acelera la desnitrificación en el Golfo Dulce en un $26 \%$. Debido a que se predice que las zonas de mínimo oxígeno se expandirán globalmente debido al cambio climático (Stramma, Johnson, Sprintall, \& Mohrholz, 2008). Por lo tanto, es indispensable monitorear la actividad microbiana anaeróbica del Golfo Dulce y su efecto ecológico en el ecosistema.

Golfo de Papagayo: Bahía Culebra: Los estudios de zooplancton de Bahía Culebra en el Golfo de Papagayo se han centrado en la composición, abundancia y biomasa del macrozooplancton y mesozooplacton marino, con énfasis en copépodos y medusas. Bednarski \& Morales-Ramírez (2004) estudiaron el macrozooplancton en cuatro sitios dentro y fuera de Bahía Culebra durante la época seca (febrero a mayo) y época lluviosa (setiembre a noviembre) del año 2000. Las mayores abundancias de macrozooplancton se obtuvieron en la época seca (febrero - marzo, 54286 ind. $/ \mathrm{m}^{3}$ ) y las menores en la época lluviosa (sep - nov, 9813 ind. $\left./ \mathrm{m}^{3}\right)$. Los copépodos (23-31\%) y los ostrácodos (20-34 \%) fueron los grupos taxonómicos dominantes en ambas épocas del año. Los 
copépodos tuvieron una dominancia significativa durante la época seca en todos los sitios de muestreo y los ostrácodos dominaron en abundancia en los sitios más externos. Este patrón temporal y espacial se asocia con eventos de surgencia costera en el Golfo de Papagayo que ocurren durante la época seca (Alfaro \& Cortés, 2012). Los copépodos de la familia Eucalanidae típicamente dominaron la abundancia en las zonas de surgencia costera (Peterson, 1988). Rodríguez-Sáenz y Morales-Ramírez (2012) reportaron los patrones anuales de la composición, abundancia y distribución espacial de 26 grupos de mesozooplacton en Bahía Culebra durante 1999-2000. Los copépodos, ostrácodos y los huevos de invertebrados fueron los grupos taxonómicos dominantes en la bahía. $\mathrm{La}$ abundancia promedio para toda la asociación del zooplancton fluctuó entre 6216 y 3969 org. $/ \mathrm{m}^{3}$, lo cual demuestra la gran variabilidad de la abundancia de cada grupo del mesozooplancton a lo largo del año. Al comparar los dos años de estudio, se encontró que las mayores abundancias se presentaron durante 1999 para todos los grupos taxonómicos, exceptuando las larvas de crustáceos y las apendicularias. La abundancia de mesozooplancton fue mayor en la época seca que en la lluviosa en ambos años asociado con los eventos de surgencia costera (Bednarski \& Morales-Ramírez, 2004). Los grupos taxonómicos holozooplanctónicos se encontraron en los sitios de muestreo más lejanos a la costa, mientras que las formas merozooplanctónicas se observaron con mayor abundancia en la parte interna de la bahía.

Rodríguez-Saénz, Vargas-Zamora, \& Segura-Puertas (2012) estudiaron la composición y la variabilidad de la abundancia de hidromedusas y registraron 53 especies. Liriope tetraphylla (Chamisso \& Eysenhardt, 1821), Solmundella bitentaculata (Quoy \& Gaimard, 1833) and Aglaura hemistoma (Péron \& Lesueur, 1810), fueron las especies más abundantes ( $>30 \%$ de la abundancia total). Seis especies se presentaron en todo el periodo de muestreo, diez se presentaron sólo durante la época seca (Dic.-Abr.) y 17 especies sólo durante la época lluviosa (May.-Nov.). La mayor abundancia de hidromedusas se encontró durante le época de influencia de surgencias costeras que influencian el Golfo de Papagayo.

Bahía Salinas: Se realizaron muestreos de zooplancton vertical (de acuerdo a la profundidad de cada estación) en 8 estaciones ubicadas en Bahía Salinas durante 2011-2013 (MoralesRamírez, 2015). Se encontraron 33 especies de copépodos, principalmente de las familias Calanidae y Eucalanidae, 25 tipos diferentes de larvas de invertebrados y 16 especies de zooplancton gelatinoso, incluidos quetognatos, cnidarios, ctenóforos y moluscos (MoralesRamírez, 2015). En este estudio se realizó en el segundo registro de la escifomedusa mesopelagica Stellamedusa ventana (Raskoff \& Matsumoto, 2004) en el Pacífico Este Tropical (Corrales-Ugalde \& Morales-Ramírez, 2017). Se encontraron en menor abundancia poliquetos planctónicos, picnogónidos, cladóceros, ostrácodos, anfípodos hipéridos y pterópodos.

La abundancia total en Bahía Salinas incrementa durante los periodos de surgencia costera semejante a lo observado en el zooplancton de Bahía Culebra (Bednarski \& Morales-Ramírez, 2004; Rodríguez-Saénz \& Morales-Ramírez, 2012) y en el Golfo de Santa Elena (MoralesRamírez, Datos sin publicar). Dichas abundancias son las mayores registradas en todos los sitios de muestreo en el Pacífico de Costa Rica, incluida la Isla Coco, donde el valor máximo reportado para la bahía fue de $>61000 \mathrm{org} . / \mathrm{m}^{3}$ (febrero 2012) y el valor mínimo fue de 1649 org. $/ \mathrm{m}^{3}$ (agosto 2011). Una agregación del dinoflagelado Noctiluca sp. causó el incremento del zooplancton más notorio en abril 2013 (120000 org. $/ \mathrm{m}^{3}$ ) durante la fase terminal del proceso de surgencia costera. En junio y agosto 2011 se recolectaron altas concentraciones de zooplancton (Cuadro 2, Morales-Ramírez, 2015). Los copépodos fueron el grupo dominante en la abundancia durante todo el periodo de muestreo. La biomasa seca fue mayor durante el periodo de surgencias en febrero 2013 y abril del 2013 (86.2 mg PS/m $/ \mathrm{m}^{3}$ y $56.1 \mathrm{mg}$ PS/ $\mathrm{m}^{3}$, respectivamente), similar a lo observado en Bahía Culebra, en el Golfo de Papagayo 
(Bednarski \& Morales-Ramírez, 2004). Los menores valores de biomasa se obtuvieron durante agosto 2012 y 2013 (18.4 y $23.2 \mathrm{mg}$ $\mathrm{PS} / \mathrm{m}^{3}$, respectivamente).

Parque Nacional Isla del Coco: El Parque Nacional Isla del Coco presenta una alta biodiversidad marina (Morales-Ramírez, 2008; Cortés, 2012; Azofeifa-Solano et al., 2016). El primer trabajo del zooplancton en la Isla del Coco reporta 136 especies del zooplancton (68 especies de copépodos, 20 especies de anfípodos y 36 géneros de ictioplancton) (Morales-Ramírez, 2008). La biomasa seca del mesozooplancton durante 2010 y 2012 varió de 77.0 a $44.9 \mathrm{mg} \mathrm{PS} / \mathrm{m}^{3}$ y del macrozooplancton varió de 28.9 a $14.0 \mathrm{mgPS} / \mathrm{m}^{3}$. Las abundancias de mesozooplancton fue de 150 ind. $/ \mathrm{m}^{3}$ durante el 2010 y 100 ind. $/ \mathrm{m}^{3}$ durante el 2012. La mayor abundancia promedio del macrozooplancton fue en 2012 (50 ind. $/ \mathrm{m}^{3}$ ) con la menor concentración durante 2011 (5 ind. $/ \mathrm{m}^{3}$ ) (Morales-Ramírez \& Vargas-Montero, 2014). Esquivel-Garrote (2015) estudió la riqueza, biomasa y composición del zooplancton asociado a parches de arrecifes coralinos en las bahías de Chatham, Weston y Wafer identificando 107 taxones de macrozooplancton y 76 de mesozooplancton. Las mayores biomasas de mesozooplancton en bahía Chatham fueron registradas durante 2009 (41.3 mg PS/m $\left.\mathrm{m}^{3}\right)$ y $2011\left(50.0 \mathrm{mg} \mathrm{PS} / \mathrm{m}^{3}\right)$. Bahía Weston las menores biomasas ocurrieron durante 2010 (14.0) y $2012\left(5.7 \mathrm{mg} \mathrm{PS} / \mathrm{m}^{3}\right)$. Los copépodos fueron el grupo taxonómico más abundante del mesozooplancton. Las mayores abundancias promedio de copépodos se obtuvieron en bahía Chatham durante el 2009 (222.9 \pm 106.7 org./ $\mathrm{m}^{3}$ ) y en bahía Wafer durante el 2011 (183.3 \pm $\left.92.6 \mathrm{org} . / \mathrm{m}^{3}\right)$. Las menores abundancias promedio se observaron en 2012 en ambas bahías $\left(48.3 \pm 24.8\right.$ en Weston y $40.1 \pm 18.9$ org. $/ \mathrm{m}^{3}$ en Wafer). Otros grupos abundantes fueron quetognatos, apendicularias, ictioplancton, larvas de decápodos y eufaúsidos.

\section{Estudios en la costa del Caribe de Costa Rica}

Caribe Sur: Los estudios de zooplancton a lo largo del litoral Caribe de Costa Rica han sido escasos en comparación con los realizados en el Pacífico, a pesar de que presenta una mayor riqueza de especies marinas por $\mathrm{km}$ de línea costera $\mathrm{o} \mathrm{km}^{2}$ de plataforma continental (Wehrtmann, Cortés, \& Echeverría-Sáenz, 2009). El primer estudio fue realizado durante 1984 en el arrecife del Parque Nacional Cahuita (Morales-Ramírez \& Murillo, 1996). No existieron diferencias significativas en abundancia durante todo el año de estudio, pero sí hubo variaciones espaciales entre las estaciones, con una mayor abundancia en una agregación en la parte somera y en el exterior de la cresta externa (Morales-Ramírez \& Murillo, 1996; Cuadro 1). Carrillo-Baltodano \& Morales-Ramírez (2016) muestrearon las mismas estaciones de zooplancton durante 2010 y 2011 reportando por primera información de biomasa zooplanctónica. Ambos estudios mostraron sorprendentes diferencias en la abundancia de zooplancton. La abundancia promedio registrada entre enero y noviembre 1984 fue considerablemente menor $\left(645 \pm 84 \mathrm{ind} . / \mathrm{m}^{3}\right)$ que durante 2010 y $2011\left(13184 \pm 41014\right.$ org. $\left./ \mathrm{m}^{3}\right)$ (Morales-Ramírez \& Murillo, 1996). Estas diferencias se debieron principalmente al incremento en abundancia de copépodos y apendicularias sugiriendo un incremento estacional de la productividad primaria de este sistema (CarrilloBaltodano \& Morales-Ramírez, 2016). Esta eutrofización pudo haber iniciado y prolongado por la continua descarga de nutrientes y sedimentos mediante la escorrentía causados por cambios en el uso del suelo en las cuencas del río Banano y el río La Estrella en los últimos 25 años (Cortés \& Risk, 1985; Mora-Cordero, 2009; Roder, Cortés, Jiménez, \& Lara, 2009). Carrillo-Baltodano \& Morales-Ramírez (2016) propusieron que esta elevada abundancia de zooplancton puede ser un recurso trófico 
relevante para los corales escleractíneos en Cahuita; a pesar de que el arrecife actualmente tiene un grado de degradación importante (Cortés et al., 2010). La presencia de estadios larvales de múltiples grupos de invertebrados permite inferir procesos de reclutamientos en Cahuita, favoreciendo la resiliencia del arrecife ante impactos naturales o antropogénicos (Carrillo-Baltodano, Morales-Ramírez, SibajaCordero, \& Cortés este volumen).

Diversidad del holozooplancton marino: El cuadro 3 resume el número de de especies de holozooplancton (549 especies) reportadas para Costa Rica, de las cuales el $44 \%$ (240, Morales-Ramírez, Suárez-Morales, CorralesUgalde, \& Esquivel-Garrote, 2014) corresponden a especies copépodos pelágicos, lo que refuerza el dominio numérico de este grupo en el zooplancton. Este amplio conocimiento sobre la diversidad del zooplancton en el país fue compilado por primera vez en la publicación del libro "Marine biodiversity of Costa Rica, Central America" (Wehrtmann \& Cortés, 2009). Este libro incluye información de biodiversidad de varios de los grupos taxonómicos del holozooplancton marino (Cuadro 3) como medusas (Rodríguez-Saénz \& Segura-Puertas, 2009), sifonóforos (Rodríguéz-Saénz \& Gasca, 2009), eufaúsidos (Castellanos, Suárez-Morales, \& Morales-Ramírez, 2009), anfípodos hipéridos (Gasca, 2009), copépodos (MoralesRamírez \& Suárez-Morales, 2009), gastrópodos pelágicos (Suárez-Morales, Gasca, \& Castellanos, 2009), quetognatos (Hernández-Flores, Morales-Ramírez, Suárez-Morales, 2009) y apendicularias (Castellanos, Morales-Ramírez, \& Suárez-Morales, 2009). En la década del 2000 se reporta por primera vez para el país, copépodos monstriloideos del género Cymbasoma y la descripción de una especie nueva, Cymbasoma concepcione (Suárez-Morales \& Morales-Ramírez, 2003).

Estudios posteriores han aumentado la lista de nuevas especies o nuevos registros para las aguas territoriales de Costa Rica. Suárez-Morales \& Morales-Ramírez (2009) describieron dos nuevas especies de copépodos de la familia Monstrillidae de la Isla del Coco (Cymbasoma cocoense y Montrillopsis chathamensis). Castellanos-Osorio et al. (2012) reportaron seis nuevos registros de apendicularias

CUADRO 3

Número de especies planctónicas reportadas en Costa Rica para algunos de los grupos taxonómico del zooplancton, junto con la referencia más actualizada para cada grupo

TABLE 3

Number of planktonic species reported in Costa Rica for some of the zooplankton taxonomic groups, together with the most updated reference for each group

\begin{tabular}{lccl}
\multicolumn{1}{c}{ Grupo Taxonómico } & $\begin{array}{c}\text { Número de especies } \\
\text { reportadas en Whertmann } \\
\text { \& Cortés (2009) }\end{array}$ & $\begin{array}{c}\text { Número de especies } \\
\text { reportadas hasta la fecha }\end{array}$ & \multicolumn{1}{c}{ Referencia más reciente } \\
$\begin{array}{l}\text { Euphausiacea } \\
\text { Appendicularia }\end{array}$ & 34 & 47 & Azofeifa-Solano et al. (2015) \\
Talacea & 5 & 18 & Corrales-Ugalde et al. (2017) \\
Amphipoda (Hyperida) & - & 23 & Nowaczyk (2008); Corrales-Ugalde (2014) \\
Copepoda & 34 & 38 & Gasca \& Morales-Ramírez (2012) \\
& 185 & 240 & Morales-Ramírez et al. (2014); \\
Chaetognatha & 28 & 34 & Esquivel-Garrote (2015) \\
Cnidaria-Medusozoa & 84 & 110 & Castellanos et al. (2012) \\
Cnidaria-Siphonophora & 37 & - & Rodríguez-Saénz et al. (2012) \\
Ostracoda & - & 4 & Morales-Ramírez \& Jacob (2008) \\
Gastropoda & 25 & 29 & Esquivel-Garrote (2015) \\
Polychaeta & - & 6 & Jiménez-Cueto et al. (2012) \\
\hline
\end{tabular}


para la Isla del Coco y dos para Costa Rica, así como seis nuevos registros de quetognatos para la Isla del Coco. Jiménez-Cueto et al. (2012) reportaron los primeros seis registros de poliquetos holoplanctónicos de la Isla del Coco. Esquivel-Garrote, Suárez-Morales, \& Morales-Ramírez (2015) describieron la especie nueva Pontella cocoensis (Garrote, SuárezMorales \& Morales-Ramírez, 2015). Gasca \& Morales-Ramírez (2012) reportaron ocho nuevos registros de anfípodos hipéridos para el Pacifico de Costa Rica. Por su parte, CorralesUgalde (2014) reportó 19 nuevos registros de tunicados pelágicos para aguas costarricenses. De igual manera, Esquivel-Garrote (2016) reportó 15 nuevos registros de copépodos, tres nuevos registros de Pteropoda, un nuevo registro de Heteropoda y dos nuevos registros de poliquetos holoplanctónicos para aguas del Pacífico de Costa Rica. Azofeifa-Solano et al. (2016) reportaron 13 nuevos registros de eufaúsidos para las aguas de la Isla del Coco. Suárez-Morales et al. (2013) describieron dos nuevas especies de copépodos Monstrillidae para las aguas del Parque Nacional Cahuita en el Caribe (Cymbasoma alvaroi (Suárez-Morales \& Carrillo, 2013) y Montrillopsis cahuitae (Suárez-Morales, Morales-Ramírez \& Carrillo, 2013) además del registro nuevo de Monstrilla grandis (Giesbrecht, 1891). Conforme se incremente el esfuerzo de muestreo de los diferentes ambientes costeros y oceánicos de Costa Rica seguramente se encontrarán nuevos registros de especies del zooplancton marino.

¿Cuál debe ser el futuro esfuerzo de investigación del zooplancton marino en Costa Rica?: El zooplancton marino de Costa Rica ha generado conocimiento de aspectos ecológicos descriptivos como abundancia, composición, biomasa, diversidad y distribución en diversos ambientes costeros: pequeñas áreas de afloramiento (Bahía Salinas y Bahía Culebra), el Golfo de Nicoya como estuario tropical de alta productividad biológica, la reserva absoluta protegida Isla del Caño, Bahía Coronado (bahía abierta y de amplia conexión oceánica), Golfo Dulce con un "fiordo" tropical anóxico de alta diversidad biológica y con sorprendentes procesos biogeoquímicos, el Parque Nacional de Cahuita (con arrecifes coralinos) y el Parque Nacional Isla del Coco, una isla oceánica localizada a $550 \mathrm{~km}$ de la costa Pacífica y patrimonio natural de la Humanidad (MoralesRamírez, 2008). Todos estos ambientes presentan condiciones oceanográficas diferentes con distinta composición y productividad del plancton. Es claro que el comportamiento del zooplancton marino en nuestras aguas obedece a las mismas condiciones oceanográficas que modulan el funcionamiento de cada ecosistema, donde es clara la similitud entre el Golfo Dulce (rasgos oceánicos) y la Isla del Coco, el carácter estuarino del Golfo de Nicoya y las condiciones estacionales de surgencia en el Golfo de Papagayo y Bahía Salinas. Menos conocemos de los rasgos oceanográficos del Caribe sur. El desarrollo de las investigaciones de zooplancton en Costa Rica ha avanzado desde una fase estrictamente descriptiva hacia investigaciones experimentales sobre aspectos tróficos del zooplancton para inferir relaciones funcionales de estos ecosistemas. Así las recomendaciones de los coloquios sobre investigaciones de zooplancton marino (Paffenhöffer et al., 1989; Bathmann et al., 2001) han permitido iniciar, sistematizar y avanzar en la investigación para ecosistemas de latitudes tropicales sobre aspectos como la función ecológica de especies pequeñas de copépodos (Oncaea sp., Oithona sp., Corycaeus sp) y grupos taxonómicos relativamente poco estudiados en ambientes costeros como ostrácodos (Morales-Ramírez \& Jacob, 2008). En Costa Rica es necesario cuantificar y comparar la biodiversidad de ecosistemas costeros y oceánicos, especialmente de grupos taxonómicos relativamente poco conocidos como apendicularias, ostrácodos, eufaúsidos, anfípodos hipéridos. El reconocimiento de regiones con alta biodiversidad marina como el Parque Nacional Isla del Coco, el Golfo Dulce y Bahía Culebra (Fonseca, 2006) deben ser investigados de una manera integral tomando en cuenta la oceanografía local y la dinámica trófica de cada ecosistema. Debido al impacto del cambio climático en la biodiversidad marina y en particular en grandes regiones 
de latitudes tropicales (Ramírez, Afán, Davis, \& Chiaradia, 2017), es oportuno llamar la atención hacia la intensificación de los estudios en estos ecosistemas con elevada biodiversidad. Los estudios futuros deben explorar el uso de herramientas moleculares y genética de poblaciones. Estos estudios serán pronto realizados gracias al nuevo laboratorio de Genética y Biología Molecular de Organismos Acuáticos del Centro de Investigación en Ciencias del Mar y Limnología de la Universidad de Costa Rica. Este laboratorio actualmente pretende evaluar aspectos sobre diferencias en estructura genética de especies claves entre la parte interna y externa del Golfo de Nicoya y cambios estacionales en la estructura poblacional en áreas de afloramientos costeros (Bahía Culebra, Bahía Salinas o el Golfo de Santa Elena).

Otros campos que deben estudiarse son cómo la dinámica poblacional de especies altamente abundantes interactúa con procesos de flujo de partículas con oscilación estacional en regiones de afloramiento costero. También es relevante el estudio trófico ampliado a las pesquerías, especialmente del Golfo de Nicoya. La información biológica de biomasa, abundancia, diversidad registrada en diferentes ecosistemas marinos de Costa Rica con distintas características oceanográficas permite realizar estudios que acoplen procesos físicos y biológicos. Este tipo de análisis del comportamiento de los organismos en ambientes naturales y en experimentos de laboratorio han avanzado considerablemente la comprensión de la ecología del zooplancton en diferentes regiones del mundo (Kiorböe, 2008). Futuros estudios comparativos entre distintos ecosistemas permitirá entender como las interacciones entre el ambiente y los organismos se relaciona con los cambios en parámetros biológicos a distintas escalas de espacio y tiempo (Prairie, Sutherland, Nickols, \& Kaltenberg, 2013).

Futuros estudios de monitoreo del zooplancton costero y oceánico, deben enmarcarse dentro del contexto del manejo y/o gestión integrada (Morales-Ramírez, 2013), si consideramos el acelerado crecimiento en los desarrollos inmobiliarios costeros en la costa Pacífica de nuestro país, en especial el Pacífico Norte y Central. Esto ha provocado diversos impactos en la dinámica costera, sus ecosistemas y los servicios ambientales que ofrecen los mismos (Morales-Ramírez, 2013).

Aunque existen estudios sobre el impacto de fenómenos como el ENSO, un seguimiento a corto, mediano y largo plazo permitiría conocer mejor como el zooplancton se adapta a un ambiente continuamente cambiante. Durante el IV Simposio Internacional de Producción del Zooplancton realizado en Hiroshima (Dagg, Harris, Uye, \& Valdés, 2008) se evidenció cambios sustanciales de la comunidad del zooplancton marino como efecto directo de los cambios climáticos globales. En este contexto es necesario generar información sobre el efecto de la acidificación costera y oceánica en la dinámica poblacional del zooplancton y en los procesos de transferencia energética dentro del zooplancton. Ya existe alguna información sobre la dinámica química de los carbonatos y su posible impacto en arrecifes coralinos del Pacífico Norte de nuestro país (Rixen, Jiménez, \& Cortés, 2012). También deben realizarse mayores esfuerzos por desarrollar más estudios de ecología larval e iniciar los estudios de parásitos, especialmente ectoparásitos como los copépodos e isópodos.

Futuros programas de monitoreo a largo plazo deberán realizarse para investigar los efectos de la acidificación oceánica y la dinámica trófica asociada con la pesca. De igual manera, es necesario estudios de largo plazo para ver tendencias de cambio en las comunidades planctónicas, especialmente comparando zonas bajo actividad humana en la zona costera (desarrollos turísticos, portuarios, urbanísticos) con zonas protegidas como blancos de estudio.

Todavía existen grandes carencias de información sobre la biodiversidad del zooplancton y ecología en aguas costeras y oceánicas del Caribe de Costa Rica. El poco esfuerzo de muestreo que se ha realizado en el Caribe de Costa Rica es posiblemente influenciado por el difícil acceso terrestre a numerosas playas ubicadas en el Caribe norte. La alta dinámica de oleaje en esta parte de Costa Rica ha 
complicado el acceso de embarcaciones con equipo científico. Además el Caribe de Costa Rica posee sólo la mitad de Áreas Marinas Protegidas del país. Esto limita el acceso a los ecosistemas marinos del Caribe, dado a que muchas de los estudios que ha realizado el CIMAR se han llevado gracias a la colaboración en logística y apoyo con miembros del Sistema Nacional de Áreas de Conservación (SINAC-MINAET).

Es evidente que buscar cooperación con instituciones que estén interesadas en explorar el Caribe centroamericano puede traer grandes beneficios para el entendimiento del zooplancton del Caribe costarricense. El fortalecimiento de las colaboraciones entre instituciones de Costa Rica como el CIMAR, SINAC-MINAET y el apoyo de la inversión privada acelerará el avance científico. Es necesario establecer una estación científica marina en el Caribe que promueva investigaciones nacionales e internacionales sobre zooplancton marino y la educación a jóvenes y público en general del cuidado y conservación de las comunidades marinas costeras.

\section{AGRADECIMIENTOS}

Agradecemos a Eleazar Ruiz por el apoyo en el trabajo de campo durante muchas de las campañas de recolecta de zooplancton marino, así como a numerosas personas que nos asistieron en el campo y en el laboratorio. Agradecemos al SINAC las facilidades y apoyo en nuestro trabajo en aquellos lugares bajo su administración. Las sugerencias y observaciones de dos revisores mejoraron la versión final de este trabajo.

\section{RESUMEN}

Este trabajo sintetiza la investigación del zooplancton marino realizada en los ambientes costeros del Pacífico y Caribe de Costa Rica, así como en el Parque Nacional de la Isla de Cocos. La composición, abundancia, biomasa, distribución y algunos aspectos ecológicos del zooplancton en cada uno de los sistemas son discutidos. La composición del zooplancton en la costa Pacífica ha sido descrito en detalle en bahía Salinas y bahía Culebra. La oceanografía local de dichas bahías está fuertemente influenciada por la corriente de vientos Papagayo, la cual produce surgencias costeras que provocan cambios estacionales en abundancia y biomasa del zooplancton. En Bahía Culebra, en el Golfo de Papagayo, los copépodos y los ostrácodos fueron los grupos taxonómicos dominantes en todo el año. Se han logrado registrar 53 especies, donde Liriope tetraphylla (Chamisso \& Eysenhardt, 1821), Solmundella bitentaculata (Quoy \& Gaimard, 1833) and Aglaura hemistoma (Péron \& Lesueur, 1810), fueron las especies más abundantes. Las tasas de herbivoría estimadas en el estuario del Golfo de Nicoya son responsables de más del $50 \%$ del consumo de la producción primaria, lo cual es una tasa que se ha estimado para otros estuarios tropicales. La comunidad de zooplancton de este Golfo está dominada. Los copépodos holoplanctónicos dominan numéricamente la comunidad del zooplancton del Golfo de Nicoya. Otros grupos abundantes del merozooplancton para este golfo son las larvas de decápodos y el ictioplancton. La biomasa es alta y hay fuertes diferencias entre las regiones interna y externa del Golfo. El zooplancton recolectado en la costa sur del Pacífico cerca de la isla de Caño está dominado por copépodos mientras que en bahía de Coronado tiene una composición mixta de zooplancton de afinidad oceánica y de transición costera. El zooplancton es altamente diverso en el Golfo Dulce, numéricamente dominado por copépodos, apendicularias y ostrácodos. E1 $35 \%$ de la producción primaria diaria es consumida por microzooplancton herbívoro. El zooplancton del Golfo Dulce parece responder a condiciones de calentamiento como el observado durante El Niño-Oscilación del Sur (ENSO). El Parque Nacional Isla del Coco es una isla oceánica del Pacífico tropical oriental, representa un ecosistema de elevada diversidad de zooplancton. Ahí se han reportado $>160$ especies de zooplancton (predominantemente copépodos). La biomasa de la Isla del Coco está dominada por mesozooplancton, y nuevos registros de especies para el Pacífico Este Tropical han sido encontrados en este ecosistema insular. Por otra parte, los primeros estudios de zooplancton en el Caribe (Parque Nacional Cahuita) en 1980 mostraron baja abundancia y diversidad de zooplancton, mientras que investigaciones realizadas finales de la década de 2000 registraron un incremento significativo en la diversidad del zooplancton, especialmente de larvas de peces Los estudios recientes del zooplancton marino han adicionado seis nuevas especies de copépodos, así como nuevos registros para la región de especies de diferentes grupos como apendicularias, anfípodos, quetognatos, eufáusidos, gastrópodos y poliquetos. Futuros programas de monitoreo a largo plazo deberán realizarse para investigar los efectos de la acidificación oceánica y la dinámica trófica asociada con la pesca. Es necesario estudios de largo plazo para ver tendencias de cambio en las comunidades planctónicas, especialmente comparando zonas bajo actividad humana en la zona costera (desarrollos turísticos, portuarios, urbanísticos) con zonas protegidas como blancos de estudio. Es recomendable aumentar los esfuerzos de muestreo en el Caribe, especialmente costa del Caribe Norte. 
Palabras clave: abundancia, biomasa, ecología, migración vertical, composición, herbivoría, Pacífico Este Tropical

\section{REFERENCIAS}

Alfaro, E., \& Cortéz, J. (2012). Atmospheric forcing of cool subsurface water events in Bahía Culebra, Gulf of Papagayo, Costa Rica. Revista de Biología Tropical , 60(2), 173-186

Azofeifa-Solano, J. C., Corrales-Ugalde, M., CastellanosOsorio, I. \& Morales-Ramírez, A. (2016). Euphausiids (Crustacea: Euphausiacea) from adjacent waters to Cocos Island, Cocos Island Marine Conservation Area, Pacific of Costa Rica. Revista de Biología Tropical , 64(Supl. 1), 221-230.

Bathmann, U., Bundy, M. H., Clarke, M. E., Cowles, T. J., Daly, K., Dam, H. G. ... Wishner, K. F. (2001). Future marine zooplankton research - a perspective. Marine Zooplankton Colloquium 2. Marine Ecology Progress Series, 222, 297-308

Bednarski, M. 2001. Macrozooplankton of Culebra Bay, Costa Rica, with an emphasis on copepods. M.Sc. Thesis, ISATEC, Universität Bremen. Bremen, Alemania. $155 \mathrm{p}$.

Bednarski, M., \& Morales-Ramírez, A. (2004). Composition, abundance and distribution of macrozooplankton in Culebra Bay, Gulf of Papagayo, Pacific coast of Costa Rica and its value as bioindicators of pollution. Revista de Biología Tropical, 53(Supl. 2), 105-118.

Brugnoli-Olivera, E., \& Morales-Ramírez, A. (2008). An aproximation on the grazing pressure of three species of copepods and their influence in the natural phytoplankton populations structure in Gulf of Nicoya, a tropical estuary, Pacific coast of Costa Rica. Revista Biología Marina y Oceanografía, 43, 75-89.

Brugnoli-Olivera, E., Díaz-Ferguson, E., Delfino-Machin, M., Morales-Ramírez, A., \& Dominici-Arosanema, A. (2004). Composition of the zooplankton community, with emphasis in copepods, in the Punta Morales zone, Golfo de Nicoya, Costa Rica. Revista de Biología Tropical, 52, 897-902.

Carrillo Baltodano, A.M. 2012. Diversidad, abundancia, composición y biomasa del zooplancton de la zona arrecifal del Parque Nacional Cahuita, Limón ¿Cuál es la disponibilidad de larvas de invertebrados bentónicos 25 años después? Tesis de Licenciatura en Biología, Universidad de Costa Rica. San Pedro, Costa Rica. 75 p.

Carrillo-Baltodano, A. M., \& Morales-Ramírez, A. (2016). Changes in abundance and community composition of coral reef zooplankton after 25 years in a Caribbean reef. Revista de Biología Tropical, 64(3), 1029-1040.
Carrillo-Baltodano, A. M., Morales-Ramírez, A. V., SibajaCordero, J. A., \& Cortés, J. (2018). New records of marine zooplanktonic invertebrates from the Caribbean coast of Costa Rica. Revista de Biología Tropical, 66(Suppl. 1), XXX-XXX.

Castellanos-Osorio, I., Hernández-Flores, R. M., MoralesRamírez, A., \& Corrales-Ugalde, M. (2012). Apendicularias (Urochordata) y quetognatos (Chaetognatha) del Parque Nacional Isla del Coco, Costa Rica. Revista de Biología Tropical, 60(Supl. 3), 2243-255.

Castellanos, I. A, Morales-Ramírez, A., \& Suárez-Morales, E. (2009). Appendicularians (Urochordata) of Costa Rica and adjacent zones. In I. S. Wehrtmann \& J. Cortés (Eds.). Marine Biodiversity of Costa Rica, Central America (pp. 445-452). Berlin: Springer + Business Media B.V.

Castellanos, I. A, Suárez Morales, E., \& Morales-Ramírez, A. (2009). Euphausiids (Crustacea, Euphausiacea) of Costa Rica and adjacent areas. In I. S. Wehrtmann \& J. Cortés (Eds.). Marine Biodiversity of Costa Rica, Central America (pp. 199-207). Berlin: Springer + Business Media B.V.

Cloern, J. E., Foster, S. Q., \& Kleckner, A. E. (2014). Phytoplankton primary production in the world's estuarine-coastal ecosystems. Biogeosciences, 11, $2477-2501$

Corrales-Ugalde, M. A. (2014). Taxonomía y distribución geográfica de los tunicados pelágicos en Costa Rica (Licenciatura's thesis). San José, Costa Rica: Universidad de Costa Rica.

Corrales-Ugalde, M. A., \& Morales-Ramírez, A. (2017). New record of Stellamedusa ventana Raskoff \& Matsumoto, 2004 in the Eastern Tropical Pacific. Marine Biodiversity, doi: 10.1007/s12526-017-07-0781-5.

Cortés, J., \& Risk, M. J. (1985). A reef under siltation stress, Cahuita, Costa Rica. Bulletin of Marine Science, 36, 339-356.

Cortés, J. 2012. Historia de la investigación marina en Bahía Culebra, Guanacaste, Costa Rica. Rev. Biol. Trop. 60 (Supl. 2): 19-37.

Cortés, J., Fonseca, A. C., Nivia-Ruiz, J., Nielsen-Muñoz, V., Samper-Villarreal, J., Salas, Zamora-Trejos, P. (2010). Monitoring coral reefs, seagrasses and mangroves in Costa Rica (CARICOMP). Revista de Biología Tropical, 58, 1-22.

Dagg, M. J., Harris R. P., Uye S.-I., \& Valdés, L. (2008). 4th International Zooplankton Production Symposium: Human and Climate Forcing of Zooplankton Populations. Hiroshima, Japan. ICES Journal of Marine Science, 65, (3).

Dittel, A. I., \& Epifanio, C. E. 1990. Seasonal and tidal abundance of crab larvae in a tropical mangrove 
system, Gulf of Nicoya, Costa Rica. Marine Ecology Progress Series, 65, 25-34.

Epifanio, C. E., \& Dittel, A. (1982). Comparison of dispersal of crab larvae in Delaware Bay, (USA) and the Gulf of Nicoya, Central America. In V. Kennedy (Ed.). Estuarine Comparisons (pp. 447-487). New York, USA: Academic Press.

Epifanio, C. E., \& Dittel, A. I. (1984). Seasonal abundance of Brachyuran crab larvae in a tropical estuary, Gulf of Nicoya, Costa Rica, Central America. Estuaries, 7, 501-505.

Esquivel-Garrote, O. (2015). Abundancia, diversidad, biomasa y composición del zooplancton, asociado a los parches de arrecife coralino en el Área de Conservación Marina Isla del Coco (ACMIC), Pacífico de Costa Rica (Licenciatura's thesis). San José, Costa Rica: Universidad de Costa Rica.

Esquivel-Garrote, O., E. Suárez-Morales \& A. Morales -Ramírez. 2015. A new species of Pontella (Calanoida, Pontellidae) from an oceanic island of the eastern tropical Pacific. Proc. Biol. Soc. Washington 128(2):137-151.

Esquivel-Garrote, O. (2016). Biomassa, Densidade e composição taxonômica do zooplâncton do Pacífico Tropical Leste (Master's thesis). Rio de Janeiro, Brazil: Universidade Federal de Rio de Janeiro.

Estrada, E. (2003). Zooplankton dynamics in the Golfo Dulce, Costa Rica, feeding preferences and grazing rates in main groups of microcrustaceans. (Master's thesis). Bremen, Germany: University of Bremen.

Fonseca-Escalante, A.C. 2006. Atlas marino-costero de Costa Rica. 209-219. En Nielsen-Muñoz, V. y Quesada-Alpízar, M.A. (eds). Ambientes Marino Costeros de Costa Rica. Comisión Interdisciplinaria Marino Costera de la Zona Económica Exclusiva de Costa Rica. Conservación Internacional, San José, Costa Rica. 219pp.

Gasca, R. (2009). Hyperiid Amphipods. In I. S. Wehrtmann \& J. Cortés (Eds.). Marine Biodiversity of Costa Rica, Central America (pp. 275-282). Berlin: Springer + Business Media B.V.

Gasca, R., \& Morales-Ramírez, A. (2012). Anfípodos hiperídeos (Crustacea, Peracarida) del Parque Nacional Isla del Coco, Costa Rica. Revista de Biología Tropical, 60(Supl. 3), 223-233.

Guzmán, H., \& Obando, V. (1988). Diversidad y abundancia diaria y estacional del zooplancton marino de la Isla del Caño, Costa Rica. Revista de Biología Tropical, 36(1), 139-150.

Hernández, R. M., Morales-Ramírez, A., \& Suárez-Morales, E. (2009). Chaetognaths of Costa Rica waters and adjacent zones. In I. S. Wehrtmann \& J. Cortés (Eds.). Marine Biodiversity of Costa Rica, Central
America (pp. 435-443). Berlin: Springer + Business Media B.V.

Hossfeld, B. (1996). Distribution and biomass of arrow worms in Golfo Nicoya and Golfo Dulce, Costa Rica. Revista de Biología Tropical, 44(Suppl.3), 157-172.

Hossfeld, B., Molina, H., \& Morales, A. (1994). Zooplankton. In, M. Wolff, \& J. Vargas (Eds.). RV Victor Hensen Costa Rica Expedition 1993/1994 cruise report (pp. 42-53). Bremen, Germany: Center for Tropical Marine Ecology.

Jacob, J. (1996). Zur Okologie und Biometrie pelagischer Ostracoden im Golfo Dulce (Costa Rica) (Master's thesis). Berlin, Germany: Freie Universität Berlin.

Jiménez-Cueto, S., Suárez-Morales E., \& Morales-Ramírez, A (2012). Algunos poliquetos holoplanctónicos (Annelida, Polychaeta) del Parque Nacional de Isla del Coco, Costa Rica. Revista de Biología Tropical, 60(Supl. 3), 207-222.

Kiorböe, T. (2008). A Mechanistic approach to plankton ecology. New Jersey, USA: Princeton University Press.

Maurer, D., \& Vargas J. A. (1984). Diversity of soft-bottom benthos in a tropical estuary, Gulf of Nicoya, Costa Rica. Marine Biology, 81, 97-106.

Mittelbach, G. G., Steiner, C. F., Schneiner, S. M., Gross, K. L., Reynolds, H. L., Waide, R. W., Gough L. (2001). What is observed relationship between species richness and productivity? Ecology, 82, 2381-2396.

Mora-Cordero, C. (2009). Factores que afecten la cuenca del Río La Estrella y recomendaciones para mejorar la gestión en la zona costera. Limón, Costa Rica (Master's thesis). San Pedro, Costa Rica: Universidad de Costa Rica.

Morales-Ramírez, A. (1987). Caracterización del zooplancton del arrecife en el Parque Nacional Cahuita, Limón, Costa Rica (Tesis de Maestría). San Pedro, Costa Rica: Universidad de Costa Rica.

Morales-Ramírez, A. (1996). Checklist of species of copepods of Gulf of Nicoya, Coronado bay and Golfo Dulce, pacific coast of Costa Rica, with comments on their distribution. Revista de Biología Tropical, 44(Supl. 3), 103-114.

Morales-Ramírez, A. (1997). Estudios del zooplancton marino. Cap. III. En J.A. Vargas Z. (ed). Estudio que identifica la condición ambiental del ecosistema, grado de impacto sobre los componentes biológicos, potencial de capacidad regenerativa y acciones de rehabilitación (Informe Final, pp. 33-66). San Pedro Montes de oca, Costa Rica. Proyecto Regional GEF/ RLA//G41 Planificación y Manejo de bahías y Áreas Costeras fuertemente contaminadas del Gran Caribe, Fondo para el Medio Ambiente Mundial, PNUD. 
Morales-Ramírez, A. (2001). Biodiversidad marina de Costa Rica, los microcrustáceos, Subbclase Copepoda (Crustacea, Maxilopoda). Revista de Biología Tropical, 49(Supl. 2), 115-133.

Morales-Ramírez, A. (2008). Caracterización cualitativa del zooplancton del Área de Conservación Marina Isla del Coco (ACMIC), Pacífico de Costa Rica. Revista de Biología Tropical, 56(Suppl. 2), 159-169.

Morales-Ramírez, A. (2013). Un análisis sobre la situación de algunos de nuestros ecosistemas costeros, necesidad de una gestión integrada. Ambientico, 230-231, $16-26$.

Morales-Ramírez, A \& M. Vargas-Montero. (2014). El plancton asociado a aguas circunvecinas y arrecifes coralinos de la Isla del Coco, diversidad, composición, abundancia, biomasa y distribución (Informe Final, proyecto 808-B0-060). San José, Costa Rica: Vicerrectoría de Investigación, Universidad de Costa Rica. Recuperado de http://hdl.handle. net/10669/74284

Morales-Ramírez, A. (2015). Composición, abundancia, biomasa y diversidad del zooplancton en Bahía Salinas, Pacífico Norte de Costa Rica (Informe final, proyecto 808-B1-194). San José, Costa Rica: Vicerrectoría de Investigación, Universidad de Costa Rica. Recuperado de http://hdl.handle.net/10669/74285

Morales-Ramírez, A., \& Vargas J. A. (1995). Especies comunes de copépodos (Crustacea) pelágicos del Golfo de Nicoya, Costa Rica. Revista de Biología Tropical, 43(1-3), 207-218.

Morales-Ramírez, A., \& Murillo, M. M. (1996). Distribution, abundance and composition coral reef zooplankton, Cahuita National Park, Limon, Costa Rica. Revista de Biología Tropical, 44, 619-630.

Morales-Ramírez, A., \& Brugnoli-Olivera, E. (2001). El Niño 1997-1998 impact on the plankton dynamics in the Gulf of Nicoya, pacific coast of Costa Rica. Revista de Biología Tropical, 49(Supl. 2), 103 -114.

Morales-Ramírez, A., \& Nowaczyk, J. (2006). El zooplancton gelatinoso del Golfo Dulce, Pacífico de Costa Rica, durante la transición de la estación lluviosa a la seca 1997-1998. Revista de Biología Tropical, 54(Supl. 1), 201-223.

Morales-Ramírez, A., \& Jacob, J. (2008). Seasonal vertical distribution, abundance and biometrical relationship of ostracods in Golfo Dulce, pacific coast of Costa Rica. Revista de Biología Tropical, 56(Supl.4), 125-147.

Morales-Ramírez, A., \& Suárez-Morales, E. (2009). Copepods. In I. S. Wehrtmann \& J. Cortés (Eds.). Marine Biodiversity of Costa Rica, Central America (pp. 291305). Berlin: Springer + Business Media B.V.
Morales-Ramírez, A., Suárez-Morales, E., Corrales, M., \& Esquivel-Garrote, O. (2014). Diversity of the freeliving marine and freswater Copepoda (Crustacea) in Costa Rica, a review. ZooKeys, 457, 15-33.

Morales-Ramírez, A., Acuña-González, J., Lizano, O., Alfaro, E., \& Gómez, E. (2015). Rasgos oceanográficos en el Golfo Dulce, Pacífico de Costa Rica, una revisión para la toma de decisiones en conservación marina. Revista de Biología Tropical, 63(Suppl. 1), 131-160.

Nowaczyk, J. (1998). Gelatinöses zooplankton in Golfo Dulce (Costa Rica), während des Übergonges von der Regen-zur Trockenzeit 1997-1998 (Master's thesis), Bremen, Germany: Universität Bremen.

Paffenhöfer, G. A, Huntley, M. E., \& Davis, C. S. (1989). Future marine zooplankton research - a perspective. Marine Zooplankton Colloquium 1. Marine Ecology Progress Series, 55, 197-305.

Peterson, W. T. (1998). Life cycle strategies of copepods in coastal upwelling zones. Journal of Marine Systems, $15,313-326$.

Pfister, A. B., \& B. Schmith. (2002). Diversity depend production can decrease the stability of the ecosystem functioning. Nature, 416, 84-86.

Prairie J. C., Sutherland, K. R., Nickols K. J., \& Kaltenberg, A. L. (2012). Biophysical interactions in the plankton, a cross-scale review. Limnology and Oceanography, 2, 121-145.

Quesada-Alpízar, M. \& Morales-Ramírez, A. (2004). Comportamiento de las masas de agua en el Golfo Dulce durante un periodo El Niño (1997-1998). Revista de Biología Tropical, 53(Supl. 2), 95-103.

Quesada-Alpízar, M., \& Morales-Ramírez, A. (2006). Posible efecto de El Niño en el zooplancton no gelatinoso de Golfo Dulce, Pacífico de Costa Rica 1997-1998. Revista de Biología Tropical, 54(Supl. 1), 225-240.

Quesada-Alpízar, M. A. (2001). Caracterización, composición, abundancia y biomasa del zooplancton en el Golfo Dulce durante el período 1997-1998. (Master's thesis). San Pedro, Costa Rica: Universidad de Costa Rica.

Ramírez, F., Afán, I., Davis, L. S., \& Chiaradia, A. (2017). Climate impacts on global hot spots of marine biodiversity. Science Advances, 3(2), e1601198. doi: 10.1126/sciadv. 1601198

Rixen, T., Jiménez, C., \& Cortés, J. (2012). Impact of upwelling events on the sea water chemistry in the Gulf of Papagayo (Culebra Bay), Costa Rica. Revista de Biología Tropical, 60(Suppl. 2), 187-195.

Roder, C., Cortés, J., Jiménez C., \& Lara, R. (2009). Riverine input of particulate material and inorganic nutrients to a coastal reef ecosystem at the Caribbean 
coast of Costa Rica. Marine Pollution Bulletin, 58, 1937-1943.

Rodríguez Sáenz, K.E. 2005. Distribución espacial y temporal de la biomasa, composición y abundancia del zooplancton, con énfasis en hidromedusas de Bahía Culebra, durante La Niña 1999 y el 2000. Tesis de Maestría en Biología, Universidad de Costa Rica. San Pedro, Costa Rica. 156 p.

Rodríguez-Saénz, K., \& Segura-Puertas, L. (2009). Hydrozoa, Scyphozoa and Cubozoa (Medusozoa). Pp., 143149. In I. S. Wehrtmann \& J. Cortés (Eds.). Marine Biodiversity of Costa Rica, Central America (pp. 143-149). Berlin: Springer + Business Media B.V.

Rodríguez-Saénz, K., \& Gasca, R. (2009). Siphonophora. In I. S. Wehrtmann \& J. Cortés (Eds.). Marine Biodiversity of Costa Rica, Central America (pp. 151-156). Berlin: Springer + Business Media B.V.

Rodríguez-Saénz, K., Vargas-Zamora, J. A. \& SeguraPuertas, L. (2012). Medusas (Cnidaria,Hydrozoa) de una zona de afloramiento costero, Bahía Culebra, Pacífico, Costa Rica. Revista de Biología Tropical, 60(4), 1731-1748

Rodríguez-Saénz, K., \& Morales-Ramírez, A. (2012). Composición y distribución del mesozooplancton en una zona de afloramiento costero (Bahía Culebra, Pacífico Norte de Costa Rica) durante La Niña 1999 y el 2000. Revista de Biología Tropical, 60(Supl. 2), 143-157.

Stief, P, Lungaard, A. S. B., Morales-Ramírez, A., Thamdrup, B., \& Glud, R. N. (2017). Fixed Nitrogen loss associated with sinking zooplankton carcasses in a coastal oxygen minimum zone. Frontiers in Marine Science, 4(152), doi: 10.3389/fmars.2017.00152

Stramma, L., Johnson, G. C., Sprintall, J., \& Mohrholz, V. (2008). Expanding oxygen-minimum zones in the tropical oceans. Science, 320(5876), 655-658.

Suárez-Morales, E., \& Morales-Ramírez, A. (2001). Nuevo registro de Acartia (Planktacartia) negligens (Copepoda, Calanoida) en el Pacífico Tropical Oriental. Revista de Biología Tropical, 40(3/4), 1286.

Suárez-Morales, E., \& Morales-Ramírez, A. (2003) A new species of Cymbasoma (Copepoda, Monstrilloida) from the Pacific coast of Costa Rica, Central America. Proceeding Biological Washington Society, 116, 206-214.

Suárez-Morales, E., \& Morales-Ramírez, A. (2009). New species of Monstrilloida (Crustacea, Copepoda) from the Eastern Tropical Pacific. Journal Natural History, $43,1257-1271$.
Suárez-Morales, E. \& R. Gasca. 2012. A new Lepeophtheirus (Copepoda: Siphonostomatoida: Caligidae) from Isla del Coco National Park, Costa Rica, Eastern Tropical Pacific. Rev. Biol. Trop. 60 (Suppl. 3): 235-242.

Suárez-Morales, E., Gasca, R., \& Castellanos, I. (2009). Pelagic gastropods. In I. S. Wehrtmann \& J. Cortés (Eds.). Marine Biodiversity of Costa Rica, Central America (pp. 357-369). Berlin: Springer + Business Media B.V.

Suárez-Morales, E., A. Carrillo \& A. Morales-Ramírez. 2013. Report on some monstrilloids (Crustacea: Copepoda) from a reef area off the Caribbean coast of Costa Rica, Central America with description of two new species. Journal of Natural History 47:619-638.

von Wangelin \& Wolff, M. (1996). Comparative biomass sprectra and species composition of zooplankton communities of Golfo Dulce and Golfo Nicoya, Pacific coast of Costa Rica. Revista de Biología Tropical, 44(Suppl. 1), 135-155.

Voorhis, A., Epifanio, C. E., Maurer, D., Dittel, A. I., \& Vargas, J. A. (1983). The estuarine character of the Gulf of Nicoya, an embayment on the Pacific coast of Central America. Hydrobiologia, 99, 225-237.

Wehrtmann, I., \& Dittel, A. I. (1990). Utilization of floating mangrove leaves as a transport mechanism of estuarine organisms, with emphasis on decapod Crustacea. Marine Ecology Progress Series, 60, 67-73.

Wehrtmann, I. S., \& Cortés, J. (2009). Marine Biodiversity of Costa Rica, Central America. Berlin: Springer + Business Media B.V.

Wehrtmann, I. S., Cortés, J., \& Echeverría-Sáenz, S. (2009). Marine biodiversity of Costa Rica, Perspectives and conclusions. In I. S. Wehrtmann \& J. Cortés (Eds.). Marine Biodiversity of Costa Rica, Central America (pp. 521-533). Berlin: Springer + Business Media B.V.

Williams, R. (1991). Spatial heterogeneity and niche differentiation in oceanic zooplankton. Hydrobiology, $167 / 168,151-160$

Wolff, M., \& Vargas, J. A. (1994). R. V. Victor Hensen Costa Rica Expedition 1993/1994. Cruise Report (Contribution 2). Bremen, Germany: Center for Tropical Marine Ecology (ZMT).

Zhang X, HG Dam, JR White \& MR Roman. 1995. Latitudinal variations in mesozooplankton grazing and metabolism in the central tropical Pacific during the U.S.JGOFS EqPac study. Deep-Sea Research 42: 695-715. 